\title{
GESTIÓN DEL CONOCIMIENTO Y CAPITAL INTELECTUAL EN LA INDUSTRIA TEQUILERA DEL ESTADO DE JALISCO
}

\author{
Marco Alberto Núñez Ramírez ${ }^{1}$
}

Recibido: 14/01/2014

Aprobado: 30/04/2014

\begin{abstract}
RESUMEN
Estudios muestran que la gestión del conocimiento se asocia con el capital intelectual; sin embargo, no se tiene claro cómo se da dicha relación. El objetivo de la presente investigación es conocer el grado de asociación de la gestión del conocimiento y el capital intelectual. Se obtuvo como evidencia empírica 50 empresas tequileras del estado de Jalisco, México. Se encontraron niveles positivos y significativos de correlación entre las mencionadas variables; así como valores significativos de influencia de la gestión del conocimiento sobre el capital intelectual, a través del empleo de variables control como el tamaño de la empresa y área de comercialización; por medio de una investigación cuantitativa y correlacional, con un diseño no experimental, donde se empleó la correlación de Pearson, regresión múltiple y regresión jerárquica.
\end{abstract}

PALABRAS CLAVE: INTANGIBLES, GESTIÓN DEL CONOCIMIENTO, CAPITAL INTELECTUAL, INDUSTRIA TEQUILERA

\section{ABSTRACT}

Studies show that knowledge management is associated with intellectual capital; however, is not clear how this relationship occurs. The objective of this research is to know the degree of association between knowledge management and intellectual capital. We obtained as empirical evidence 50 Tequila companies of the State of Jalisco, Mexico. We found positive and significant levels of correlation between the above variables; as well as significant values of influence of the knowledge management on intellectual capital, through the use of control variables such as the size of the company and area of trade, through a correlational, and quantitative research with a non-experimental design, where the correlation of Pearson, multiple regression and hierarchical regression were used.

KEYWORDS: INTANGIBLES, INTELLECTUAL CAPITAL, KNOWLEDGE MANAGEMENT, TEQUILA INDUSTRY

1 Universidad Nacional Autónoma de México; México. Código Postal 54740; mnunezram@comunidad.unam.mx 


\section{INTRODUCCIÓN}

Nuevos cambios se están produciendo en la economía mundial. Éstos llevan a considerar al conocimiento como el elemento básico de la escena empresarial (Nevado \& López, 2002). La tierra, el capital y la mano de obra siguen la ley de rendimiento decreciente, mientras que el conocimiento disfruta de un crecimiento (Roos et al, 2001). El dinero mismo se ha desmaterializado y los activos intangibles se han hecho más valiosos y poderosos que los recursos naturales, las grandes fábricas o las cuentas bancarias (Brooking, 1997a).

La llegada de la era del conocimiento es una realidad (Obeso, 2003). Ahora, los activos intangibles juegan un papel relevante en la creación de valor de las empresas (Edvinsson \& Malone, 2001). Los recursos y procesos intangibles que pertenecen a la compañía como el conocimiento tácito y explícito determinan la competitividad (Bontis, 1999).

Aunque, la primera mención del concepto de intangible en la empresa se le atribuye a Lawrence Dicksse, que data de 1896 (Wu, 2005), fue en los años noventa cuando académicos y consultores en gestión empresarial comienzan a prestarle atención (Simó \& Sallán, 2008). Para Brooking (1997a) el valor de la empresa no reside en los activos materiales, sino en los inmateriales. Constituyen uno de los principales factores de éxito presente $y$ futuro de las empresas, por lo que cada vez se incrementan más las inversiones en este tipo de activos (Nevado \& López, 2002). Ahora, el valor de una empresa depende fundamentalmente de la valoración de sus activos inmateriales (Obeso, 2003); sin embargo uno de los principales problemas cuando se habla de éstos, es determinar qué se entiende por activos intangibles.

De acuerdo con Funes (2010) los activos intangibles son aquellos identificables, sin sustancia física y fuentes de riqueza. Poseen capacidad para generar beneficios económicos futuros y pueden ser controlados por la empresa (Requena, Sellens \& Masllorens, 2003); son valiosos para la organización y se orientan a largo plazo (Edvinsson \& Malone, 2001); sin embargo, hoy en día, la mayoría de las compa- ñías desconocen su valor y cómo se gestionan (Brooking, 1997a).

Dentro de tales activos destacan los intangibles ocultos, que corresponden al capital intelectual (Nevado \& López, 2002), el cual es definido como un conjunto de recursos y capacidades inmateriales, expresiones del conocimiento individual y colectivo, que se manifiestan en capital humano, estructural y relacional (Mercado \& Cernas, 2012). Por otro lado, el conocimiento es el activo más importante que se pueda tener a lo largo de la vida, y se debe dedicar una proporción considerable del tiempo a su cuidado (Brooking, 1997a; Stewart, 1998). De tal modo que hoy en día, la organización es percibida como una institución que busca integrar el conocimiento como fuente primaria de valor (Matilla, 2000). Para lograrlo, requiere del empleo de la gestión del conocimiento, la cual es concebida como la capacidad por medio de la cual una compañía como un todo puede crear conocimiento, diseminarlo a través de la organización y encarnarlo en los productos, servicios y sistemas (Nonaka \& Takeuchi, 1995).

Cabe resaltar que la gestión del conocimiento y el capital intelectual dentro de las organizaciones no son una teoría del todo, sino una perspectiva en constante construcción. $\mathrm{Su}$ soporte teórico se encuentra tanto en la teoría de recursos y capacidades (RBV) como en la teoría basada en el conocimiento (KBV). La teoría de recursos y capacidades desde sus inicios ha considerado al conocimiento como un recurso intangible, capaz de darle a la organización una ventaja competitiva, al ser raro, valioso, imperfectamente imitable y no sustituible (Barney, 1991). De RBV se desprende la teoría basada en el conocimiento (KVB), la cual aborda a la gestión del conocimiento como elemento central para el desarrollo de capacidades en las organizaciones (Grant, 1996a; Sveiby, 2001). Para Grant (1996b) la teoría basada en el conocimiento se fundamenta en el proceso de transferencia, capacidad de absorción, apropiación, especialización y transformación del conocimiento en productos dentro de la empresa. Dicho proceso es conocido como gestión del conocimiento (Nonaka \& Takeuchi, 1995). 
Por otro lado, el tequila es un producto tradicional $y$ representativo de la cultura mexicana en el interior y exterior del país; su industria aporta una parte significativa del PIB y es fuente de empleo en varios municipios del Estado de Jalisco (Macías, 1997), donde se pueden localizar 152 empresas tequileras (CRT, 2013). Los destilados del tequila significan aportaciones importantes a la economía local y nacional (Casas-Campillo, 1999), aunque su participación en el mercado mundial de licores sólo es de $1.02 \%$, el crecimiento en las últimas décadas ha sido notable (Orozco, 2011). El tequila es una industria altamente consolidada, la cual genera casi 4,592 millones de dólares, colocándola en el segundo producto mexicano de mayor exportación del sector agroalimentario, sólo después de la cerveza, teniendo una participación del $12.2 \%$, mayor que la obtenida en el período 1991-1993, que fue de 8.2\% (Macías \& Valenzuela, 2009).

Sin embargo, la industria tequilera se encuentra orientada hacia una dependencia progresiva de los capitales extranjeros: empresas multinacionales en el caso del tequila embotellado y embotelladores estadounidenses que lo venden a granel (Coelho, 2007). La industria se encuentra caracterizada como un oligopolio (Macías, 2001), donde desde la segunda mitad del siglo XIX, algunas empresas al ser pioneras en el uso de recursos tecnológicos de la industria mundial establecieron estructuras hegemónicas, lo que les ha permitido asegurar un liderazgo. Por ello, sólo cuatro empresas controlan cerca del 65\% del mercado (Coelho \& Castillo-Giron, 2004), a pesar de que para el 29 de abril del 2013 se encontraban registradas 1,588 marcas de tequila, y 112 marcas de bebidas con tequila, que conforman un total de 1700 marcas a nivel mundial (CRT, 2013).

Según COFUPRO (2003) dentro de los principales problemas que enfrenta la industria se encuentra la transferencia tecnológica, delimitada por la pérdida del agave, carencia tecnológica, poca variedad, falta de investigación y desarrollo, medios de comunicación inadecuados, escasa generación de tecnología y proyectos de investigación. Aunado a lo anterior, la mayoría de las empresas tequileras son familiares $y$ se encuentran estancadas en técnicas ancestrales que impiden la competitividad que demanda la cadena de esta industria (Salvador \& Maldonado, 2010). Históricamente la industria ha sido marcada por conflictos $y$ dificultades a su organización (Coelho, 2007), a pesar que el posicionamiento del tequila en el mercado de bebidas alcohólicas alcanzó un 46\% (Macías \& Valenzuela, 2009), todavía existen problemas referentes estándares de calidad (Macías, 2001).

Partiendo de la denominación de origen del tequila, el conocimiento debe ser generado en el dentro de las regiones productoras de agave. Sin embargo, se considera necesario fundamentar otra filosofía en las empresas tequileras, lo cual les permita alcanzar mayor competitividad (Díaz, 2007). Como resultado de la expansión del mercado del tequila, existen diferentes programas para generar conocimientos básicos e información aplicada que permitan asegurar la producción de materia prima $y$ transferencia del conocimiento (Rulfo et al, 2007). Es por ello, que el empleo de intangibles como el capital intelectual y la gestión del conocimiento pueden resolver los problemas de competitividad que aquejan a la gran mayoría de las empresas que se enfrentan a corporativos ya consolidados que cuentan con capitales extranjeros y manejo de tecnología.

\section{Gestión del conocimiento}

El conocimiento ha sido abordado por diferentes disciplinas; sin embargo, se considera a la epistemología como la base para su estudio, donde se le define como un acto de relación establecido entre un sujeto $y$ un objeto, donde el sujeto aprehende una realidad (Verneaux, 1996). Asimismo, su concepción actual se fundamenta en una herencia de doctrinas como el empirismo, racionalismo, fenomenología, idealismo y realismo (Cortés \& Martínez, 1996). Por otro lado, el conocimiento proviene de los problemas, pues no hay conocimiento sin problemas; sus raíces se encuentran en la tensión entre el saber y la ignorancia (Popper, 1978). Éste es el sentido pragmático 
tomado por la gestión del conocimiento dentro de las organizaciones.

Se reconocen dos tipos de conocimiento en la organización: el tácito y el explícito (Nonaka, 1994). El primero tiende a ser local porque no se encuentra en manuales, libros, bases de datos ni archivos; es oral, se crea y se difunde informalmente (Stewart, 1998), es más difícil de articular porque con frecuencia supera a la experiencia, ya que el conocimiento no registrado es a menudo más rico que la memoria documentada (Obeso, 2003). Mientras el explícito es el que puede ser escrito y relativamente transferido de una persona a otra de una forma ordenada (Nonka \& Konno, 1994; Nonaka \& Takeuchi, 1995). Ambos tipos constituyen un ciclo infinito que consiste en identificar el conocimiento tácito, volverlo explícito con el fin de formalizarlo, aprenderlo y potencializarlo (Stewart, 1998).

El conocimiento no registrado es a menudo más rico que la memoria documentada (Obeso, 2003). De tal modo que surge la gestión del conocimiento como la administración explícita y sistemática del conocimiento y sus procesos asociados de creación, recolección, organización, difusión y explotación (Matilla, 2000).

De acuerdo con Rodríguez (2006) la aparición y desarrollo de los sistemas para la creación y la gestión del conocimiento se deben al cambio en el sistema socioeconómico, la aparición y desarrollo de las tecnologías de la comunicación, la relación del conocimiento con la efectividad organizacional, el fracaso de los modelos financieros tradicionales y el aumento de la competitividad entre las organizaciones.

Brooking (1997a) afirma que estudios en materia de gestión de conocimiento han revelado que en la práctica sólo se usa alrededor de un $20 \%$ de los conocimientos con los que dispone la empresa. Por ello, la completa aplicación del conocimiento es un objetivo en sí mismo $y$ significa desarrollar un nuevo conocimiento (Roos et al, 2001).

Como en la era de conocimiento la fuerza de trabajo está basada en los conocimientos (Brooking, 1997a), los cambios en la economía en los últimos años han hecho del conocimiento la fuente más importantes de la ventaja com- petitiva $y$ del éxito que puede tener una compañía, ya que éste es más valioso y poderoso que los recursos naturales, las grandes fábricas o las cuentas bancarias abultadas (Roos et al, 2001). Por lo tanto, es un activo $y$, al igual que todos los activos tiene que administrarse (Brooking, 1997b); pues competir con conocimientos es privilegio de pocas empresas (Obeso, 2003). Es así como surge el interés por la gestión del conocimiento dentro de las empresas.

Actualmente no existe una definición formalmente aceptada respecto a la gestión del conocimiento, aunque se han realizado diferentes acercamientos. La gestión del conocimiento es la función que planifica, coordina $y$ controla los flujos de conocimientos que se producen en la empresa en relación con sus actividades y con su entorno (Bueno, 199); proceso de creación, captura y uso del conocimiento para mejorar el desempeño organizacional (Edwards, Collier \& Shaw, 2005).

Es la capacidad de que una compañía como un todo pueda crear conocimiento, diseminarlo a través de la organización y encarnarlo en los productos, servicios y sistemas (Nonaka \& Takeuchi, 1995). Se dedicada a la dirección de las tácticas y estrategias requeridas para la administración de los recursos humanos intangibles en una organización (Brooking, 1997a). Es la tarea de reconocer un activo humano enterrado en las mentes de las personas y convertirlo en un activo empresarial al que puedan acceder $y$ que pueda ser utilizado por un mayor número de personas (Tejedor \& Aguirre, 1998).

En un sentido genérico, la gestión del conocimiento está conectada con el cómo los individuos aprenden dentro del contexto organizacional (Edwards, Collier \& Shaw, 2005). Depende de la cultura existente en la organización $y$, además se relaciona dinámicamente con la dirección o administración de un conjunto de flujos de conocimientos: externos o internos, captados o creados, explícitos o tácitos (Bueno, 1999; Sullivan, 2001).

La gestión del conocimiento está dedicada a la dirección de las tácticas y estrategias requeridas para la administración de los recursos humanos intangibles en una organización 
(Brooking, 1997b). Es la tarea de reconocer un activo humano enterrado en las mentes de las personas y convertirlo en uno empresarial que pueda ser utilizado por un mayor número de personas (Tejedor \& Aguirre, 1998). Se relaciona dinámicamente con la dirección o administración de un conjunto de flujos de conocimientos: externos o internos, captados o creados, explícitos o tácitos (Nonaka, 1994; Bueno, 1999).

De manera inclusiva se puede decir que la gestión del conocimiento es la disciplina que enmarca procesos $y$ funciones de suma importancia de la organización, donde se gestiona la capacidad de optimizar el proceso de generación, administración y transmisión del conocimiento, con el fin de concebir productos $y$ servicios, que a su vez conforman una ventaja competitiva sostenida dentro de la sociedad del conocimiento.

Dentro de los modelos que estudian la gestión del conocimiento el SECI propuesto por Nonaka y Takeuchi (1995), es uno de los de mayor relevancia en la actualidad. Toma su punto de partida en la concepción del conocimiento como un proceso humano dinámico, dividido en tácito y explícito (Nonaka, 1994). El conocimiento tácito es personal y difícil de formalizar, lo cual impide su comunicación con los miembros de la organización. El explícito representa la parte racional del conocimiento, el cual puede ser expresado y explicado fácilmente en palabras y números, por lo cual puede ser transmitido a otros para ser procesado colectivamente (Bratianu, 2010). Este modelo ha causado interés en diferentes investigadores como Nonaka y Konno (1994), Nonaka y Takeuchi (1995), Kogut y Zander (1996) y Mihi, García y Rojas (2011).

Para Nonaka y Takeuchi (1995) la creación del conocimiento organizacional es la capacidad de una compañía como un todo para crear conocimiento, diseminarlo a través de la organización y encarnarlo en los productos, servicios y sistemas. Asimismo, se refieren a la conversión del conocimiento, a través de la cual se pretende convertir el conocimiento tácito a explícito para que pueda ser compartido y usado por la organización con el fin de crear uno nuevo (Nonaka, Reinmoeller \& Senoo, 1998). De tal manera el conocimiento fluye de tácito a tácito mediante la adquisición, de tácito a explícito por la conversión, de explícito a explícito por la creación, y de explícito a tácito por medio de la incorporación (Angulo \& Negron, 2008).

De acuerdo a Nonaka \& Toyama (2003) el conocimiento necesita cuatro contextos para ser creado, donde se permite la conversión del conocimiento tanto individual o grupal como tácito o explícito. El modelo denominado SECI se encuentra enmarcado por cuatro etapas: socialización, externalización, combinación e internalización. La socialización (grupal tácitoindividual tácito), donde se crea una sincronización y entendimiento básico para la creación del conocimiento favoreciendo la autonomía de los empleados. La externalización (individual tácito-individual explícito) estimula la reflexión a través del diálogo, convirtiéndose en fundamenta la formación, los conocimientos individuales y la cohesión de grupo. La combinación (individual explícito-grupal explícito) se apoya en soportes tecnológicos que permiten la mezcla de diferentes conocimientos para la construcción de arquetipos (Nonaka \& Takeuchi, 1995). La internalización (grupal explícitogrupal tácito) busca la creación de entornos de trabajo para ayudar en la internalización del conocimiento por parte de los miembros de la organización (Nonaka, 1994).

\section{Capital intelectual}

Aunque el origen del estudio del capital intelectual se puede localizar en Müller (17791829) quien habló del capital científico y mental como un conjunto de competencias constructivas del hombre, Estado y sociedad, fue a finales de los años 50 e inicios de los 60 cuando se encuentran sus principales referencias (Mercado \& Cernas, 2012). Penrose (1959) vislumbra a los activos intangibles como generadores de valor en la organización, donde se puede encasillar al conocimiento. Machlup (1962) resaltó el valor económico del conocimiento; mientras que Drucker (1969) anunció el inicio de la sociedad del conocimiento. 
Fue hasta 1969 cuando John Kenneth Galbraith acuñó el concepto de capital intelectual como acción intelectual más que mero conocimiento o puro intelecto (Mercado \& Cernas, 2012). En los inicios de los años 90 este tema atrajo mayor atención dentro de los académicos surgiendo estudios como los de Kaplan y Norton (1992), Skandia (1994), Saint-Onge (1996), Bontis (1996), Sveiby (1996), Brooking (1997a), Stewart (1998), Edvinsson y Malone (2001).

Aunque no existe actualmente una definición aceptada por la comunidad especializada del capital intelectual han surgido diferentes visiones sobre este concepto. Según Brooking (1997a) el capital intelectual no es nada nuevo, es la combinación de activos inmateriales que pertenecen a la empresa. Su estudio nace con la investigación de Skandia (1994), que lo divide en humano y estructural. Se define como la posesión de conocimientos, experiencia aplicada, tecnología organizacional, relaciones con clientes $y$ destrezas profesionales que dan una ventaja competitiva en el mercado (Edvinsson \& Malone, 2001).

El capital intelectual es un término dado a todos los intangibles, los cuales permiten la función de la organización (Brooking, 1997b). Consiste en la información, propiedad intelectual, material intelectual, conocimiento $y$ experiencia. Puede ser utilizada para crear valor; aspecto que conlleva el capital humano, estructural, y relacional, teniendo una gran relevancia para la administración moderna, donde se incluyen los procesos organizacionales, tecnologías, patentes, habilidades de los empleados, información acerca de los clientes y los suministros (Stewart, 1998). Abarca las relaciones con los clientes y socios, los esfuerzos innovadores, la infraestructura de la compañía $y$ el conocimiento $y$ la pericia de los miembros de la organización (Ross et al, 2001), configurado por todo lo que hay dentro de la compañía: sus recursos, procesos intangibles, patentes, innovaciones, clientes, así como conocimiento tácito y explícito (Bontis, 1999). Su aparición es considerada como un producto de la era del conocimiento (Edvinsson \& Malone, 2001). Este es un tema que cada vez interesa más a las empresas que obtienen sus beneficios con la innovación y el conocimiento (Sullivan, 2001); sin embargo, el capital intelectual puede ser una postura nueva pero en la práctica ha existido desde hace años como cuestión de sentido común (Edvinsson, 1997).

El capital intelectual surge como la necesidad de valorar a la empresa de una forma detallada, yendo más allá de los activos visibles, hasta considerar la preponderancia de los intangibles con los que cuenta la organización para competir. Representa la fusión entre dos corrientes del pensamiento: la administración y medida del conocimiento (Roos et al, 2001), a partir de las cuales varía su conceptualización. A grandes rasgos se puede decir que el capital intelectual es un concepto sombrilla que sirve para sintetizar y evaluar aquellos recursos de la organización cuya naturaleza es intangible, el cual no es un fin para la empresa, sino un medio para competir y sobrevivir en mercados totalmente globales (Mercado \& Cernas, 2012).

Existen ciertas congruencias en cuanto a la clasificación del capital intelectual. El Navegador de Skandia (1994) consta de dos capitales: humano y estructural. Stewart (1998) y, Edvinsson y Malone (2001) mantienen de manera general tal clasificación. The Technology Broker de Brooking (1997a) clasifica los activos intangibles en mercado, humano propiedad e infraestructura. Saint-Onge (1996) añade el capital relacional a la propuesta de Skandia, conformando una taxonomía de capital intelectual constituida por capital humano, capital estructural y capital relacional, la cual ha sido retomada en un sentido general por Nevado $y$ López (2000); Bontis (1996); Bueno (1999); y el Modelo Intelect, CIC $(2002 ; 2003)$. Tal clasificación es tomada para el presente estudio.

\section{Capital humano}

El capital humano hace referencia al conjunto de capacidades de los empleados (Brooking, 1997a). De acuerdo con Edvinsson y Malone (2001) el capital humano es la base del capital intelectual; es la combinación de conocimientos, habilidades, inventiva y capacidad de los empleados para llevar a cabo tareas; conocimientos útil que poseen las personas (CIC, 2002). Conlleva capacitación, formación 
$y$ aprendizaje (Madrigal, 2009). Capital constituido por personas cuyo talento $y$ experiencias crean productos y servicios que atrae a los clientes (Stewart, 1998). Son el conjunto de conocimientos tácitos y explícitos adquiridos mediante procesos de educación, socialización reciclaje $y$ actualización propios de su actividad (Ramírez et al, 2007).

\section{Capital estructural}

El capital estructural es la infraestructura que incorpora, forma $y$ sostiene el capital humano; incluye los sistemas físicos usados para transmitir y almacenar el conocimiento; infraestructura que facilita el acceso al conocimiento (Edvinsson \& Malone, 2001). Se refiere a lo que permanece en la organización cuando los empleados se van (Skandia, 1994). Contempla los procesos internos de diseminación, comunicación y administración del conocimiento técnico y científico (Ramírez et al, 2007). El capital estructural es el conjunto de conocimientos $y$ de activos intangibles derivados de los procesos que pertenecen a la organización (CIC, 2003). Vínculo entre el capital humano y el capital relacional (Stewart, 1998)

\section{Capital relacional}

El capital relacional es el valor que representa para la organización las relaciones que ésta mantiene con sus miembros, otras organizaciones, clientes, proveedores y otros agentes sociales que actúan en su entorno. (Stewart, 1998). Se define como las relaciones que tiene la empresa con sus miembros $y$ agentes externos como los clientes, proveedores y la sociedad (CIC, 2002). De acuerdo con CIC (2003) es el conjunto de conocimiento que se incorporan a la organización y a las personas como consecuencia del valor derivado de relación de la organización con agentes del mercado y sociedad.

\section{Gestión del conocimiento y Capital intelectual}

Por su misma naturaleza existen ciertos puntos de intersección entre la gestión del conocimiento y el capital intelectual: 1) ambos son consideraros recursos importantes para la organización (Brooking, 1997a; Stewart, 1998; Barney, Ketchen \& Wright, 2011); 2) son activos intangibles difíciles de administrar (Nevado \& López, 2002); 3) buscan eficientizar el uso del conocimiento para crear valor de la empresa (Roos et al, 2001); 4) son soportados por la teoría de recursos y capacidades (Barney, 1991) y la teoría basada en el conocimiento (Grant, 1996a).

De acuerdo a Matilla (2000) existen dos formas de abordar la gestión del conocimiento: una como parte del capital intelectual $y$ otra como gestión del conocimiento propiamente dicho. Entendiendo que éste es el conjunto de actividades encaminadas a crear el capital intelectual (Nava y Mercado, 2010). Como variable perteneciente al capital intelectual es abordado por Bontis (1996), Saint-Onge (1996), Brooking (1997b), Bueno (1999), Edvinsson y Malone (2001), Roos et al (2001), Sullivan (2001), el Modelo Intellectus CIC (2002) y, Nevado y López (2002). No obstante, la riqueza del estudio de la gestión del conocimiento se encuentra al considerarlo por sí mismo. Bajo esta segunda perspectiva ésta ha sido estudiada por Nonaka y Konno (1994), Nonaka y Takeuchi (1995), Kogut and Zander (1996) Tejedor y Aguirre (1998), Andersen (1999), Obeso (2003), Edwards, Collier y Shaw (2005).

El capital intelectual representa conocimiento transformado en algo valioso para la organización, cuando dicho conocimiento es utilizado y compartido para crear valor llega a ser capital intelectual (Martínez, 2003). La gestión del conocimiento es reconocida como una actividad fundamental para obtener, acrecentar y sostener el capital intelectual (Marr \& Schiuma, 2001). Una gestión exitosa del capital intelectual se encuentra ligado al proceso de gestión del conocimiento (Marr et al, 2003). El capital intelectual representa el valor creado por el sistema que representa la gestión del conocimiento (Bueno, 1999). A partir de tal supuesto se plantea la primera pregunta de investigación: ¿Cómo se asocia la gestión del conocimiento y el capital intelectual? 
Estudios proponen que la gestión del conocimiento se asocia con el capital intelectual (Koening, 1998; Vargas, 2002; Martínez, 2003; Curado, 2008; Booker, Bontis \& Serenko, 2008; Antosova \& Csikosova, 2011; Serenko, Bontis \& Hull, 2011); sin embargo, no precisan en qué forma se da dicha relación, ni muestran evidencia empírica de ello. Salmninezhad y Daneshvar (2012) encontraron niveles positivos de correlación sólo entre los capitales estructural y relacional con la gestión del conocimiento. Se refleja con claridad la necesidad de mayor evidencia empírica que sustente tal premisa, por ello se propone como primera hipótesis:

\section{$H_{t}$ : El capital intelectual se asocia de manera positiva con la gestión del conocimiento}

Por otro lado, una implementación exitosa de la gestión del conocimiento implica la adquisición y crecimiento del capital intelectual (Marr et al, 2003). La gestión del conocimiento integra el capital humano (valor del conocimiento creado por las personas), capital estructural (conocimiento creado en la organización materializado en sistemas, procedimientos y tecnología), y capital relacional (conocimiento creado en relación con su entorno) (Bueno, 1999). Asimismo, el conocimiento tácito se manifiesta en conocimiento codificado y el explícito a través de procesos (capital humano); se combina utilizando los procesos sociales para combinar los elementos (capital estructural); se obtiene, incorpora $y$ aplica por medio de la relación con clientes, proveedores, académicos, agentes de mercado y proveedores (capital relacional) (Fierro, Mercado \& Cernas, 2012). Dichas posturas no se han sustentado empíricamente, por lo cual se plantea la segunda pregunta de investigación de la siguiente manera: ¿Cómo influye la gestión del conocimiento sobre los componentes del capital intelectual?

Tomando en cuenta que la gestión del conocimiento es un proceso mientras que el capital intelectual es su producto (Fierro, Mercado \& Cernas, 2012), se puede entender que la gestión del conocimiento influye de cierta manera sobre el capital intelectual. Dicha influencia debe de reflejarse en los componen- tes del capital intelectual, donde se patenta el conocimiento. A partir de tal premisa se presentan las siguientes hipótesis:

$\mathrm{H}_{2}$ : La gestión del conocimiento influye sobre el capital humano.

$H_{3}$ : La gestión del conocimiento influye sobre el capital estructural

$H_{4}$ : La gestión del conocimiento influye sobre el capital relacional.

\section{METODOLOGÍA}

La presente investigación es de tipo cuantitativa, y correlacional, con un diseño no experimental y transversal. En la recolección de datos primarios se emplearon dos instrumentos de medición. Para la gestión del conocimiento, se aplicó un cuestionario basado en Mihi, García y rojas (2011), el cual se centra en el modelo SECI (socialización, externalización, combinación e integración) de Nonaka y Takeuchi (1995) compuesto por 11 reactivos con una escala Likert-5, donde: $1=$ totalmente en desacuerdo, $2=$ en desacuerdo, $3=$ indeciso, $4=$ de acuerdo y 5 =totalmente de acuerdo. Para el capital intelectual se administró un cuestionario elaborado la Dra. Patricia Mercado, como resultado del Proyecto de Elaboración de un Instrumento de Medición de Capital Intelectual en la Universidad Autónoma del Estado de México (Mercado, Cernas \& Demuner, 2014). La escala fue Likert-5, con valores $1=$ muy inadecuado, $2=$ inadecuado, $3=$ regular, $4=$ adecuado $y$ $5=$ muy adecuado. A mayor calificación se refleja el desarrollo del capital humano, estructural o relacional.

Los datos fueron obtenidos mediante autoadministración y envío electrónico a través del portal www.encuesfácil.com en el período enero-junio 2013. Se obtuvo una muestra por conveniencia conformada por 50 empresas de la industria tequilera ubicadas en el Estado de Jalisco. El 28\% de la muestra se integró por microempresas, $36 \%$ pequeñas, $22 \%$ por medianas y $14 \%$ por empresas grandes, ubicadas en las regiones Valles (28.6\%), Centro 
(26.5\%), Altos Sur (18.4\%), Ciénega (8.2\%), Altos Norte (6.1\%), Sierra Occidental (4.1\%) y con $2 \%$ Sur, Sierra de Amula, Norte y otras. El $57.1 \%$ de las empresas comercializan sus productos tanto en mercados nacionales como internacionales, $28.6 \%$ nacionales y 14.3\% sólo internacionales. Respecto al puesto de la persona que contestó el cuestionario, $36.7 \%$ de los encuestados poseen un puesto gerencial, $18.4 \%$ de jefatura, el $12.2 \%$ supervisión, $8.2 \%$ coordinación $y, 4.1 \%$ tanto puestos administrativos como auxiliar de gerencia.

\section{RESULTADOS}

Se obtuvieron resultados preliminares favorables para la normalidad, correlación ítemtest, KMO, prueba de esfericidad de Blartlett, los cuales muestran la conveniencia del análisis factorial exploratorio, el cual descubre las agrupaciones de las variables de estudio. El análisis factorial exploratorio se realizó por medio de análisis de componentes principales con rotación varimax para medir la validez de constructo, la cual se refiere al grado en que un instrumento realmente mide la variable que pretende medir (Montoya, 2007). Se encontraron tres componentes para capital intelectual y cuatro en el caso de la gestión del conocimiento. Los tres capitales: humano, estructural y relacional explicaron el $75 \%$ de la varianza, mientras que la socialización, externalización, combinación e internalización el 93\%.

Se verificó la confiabilidad, la cual se refiere al grado de consistencia de un instrumento, cuyos valores alpha de Cronbach deben ser $\geq 0.7$ (Hair, Anderson \& Black, 2004). Los resultados de confiabilidad fueron favorables para los componentes tanto de gestión del conocimiento como de capital intelectual: socialización $(\alpha=0.94)$, externalización $(\alpha=0.94)$, combinación $(\alpha=0.95)$, internalización $(\alpha=0.80)$, capital humano $(\alpha=0.97), \quad$ capital estructural $(\alpha=0.95)$ y capital relacional $(\alpha=0.94)$ (Tabla 1). Los resultados descriptivos mostraron valores similares en cuanto a las medias: socialización $(\tilde{\chi}=3.87 ; \sigma=0.78)$, externalización $(\tilde{\chi}=3.28 ; \sigma=1.13)$, combinación $(\tilde{\chi}=3.5 ; \sigma=1.07)$, internalización $(\tilde{\chi}=3.8 ; \sigma=0.92)$ capital humano $(\tilde{\chi}=3.82 ; \sigma=0.87)$, capital estructural $(\tilde{\chi}=3.62 ; \sigma=0.94)$ y capital relacional $(\tilde{\chi}=3.87$; $\sigma=0.95)$ (Cuadro 1).

\section{CUADRO 1 \\ MEDIA, DESVIACIÓN ESTÁNDAR Y CONFIABILIDAD}

\begin{tabular}{cccccc}
\hline Variable & Componente & Ítems & $\tilde{\chi}$ & $\sigma$ & Confiabilidad \\
\hline \multirow{2}{*}{$\begin{array}{c}\text { Gestión del } \\
\text { Conocimiento }\end{array}$} & Socialización & 3 & 3.87 & 0.78 & 0.94 \\
& Externalización & 3 & 3.28 & 1.13 & 0.94 \\
& Combinación & 3 & 3.5 & 1.07 & 0.95 \\
& Internalización & 2 & 3.8 & 0.92 & 0.80 \\
Capital intelectual & Capital humano & 15 & 3.82 & 0.87 & 0.97 \\
& Capital estructural & 11 & 3.62 & 0.94 & 0.95 \\
& Capital relacional & 12 & 3.87 & 0.95 & 0.94 \\
\hline
\end{tabular}

Fuente: Elaboración propia.

Se utilizó $r$ de Pearson para poner a prueba la hipótesis 1 , referente a la asociación de variables de estudio. Todos los componentes de las variables se correlacionaron con valores significativos $y$ positivos. La socialización alcanzó valores altos respecto al capital humano $(r=0.835 ; \mathrm{p} \leq 0.01)$, capital estructural $(r=0.815 ; \mathrm{p} \leq 0.01)$ y capital relacional $(r=0.693$; 
$\mathrm{p} \leq 0.01)$. Lo mismo sucedió en la externalización respecto al capital humano $(r=0.857$; $\mathrm{p} \leq 0.01)$, capital estructural $(r=0.867 ; \mathrm{p} \leq 0.01)$ y capital relacional $(r=0.830 ; \mathrm{p} \leq 0.01)$. La asociación para la combinación fue de mediano a alto en relación con capital humano $(r=0.769$; $\mathrm{p} \leq 0.01)$, capital estructural $(r=0.837 ; \mathrm{p} \leq 0.01)$ y capital relacional $(r=0.722 ; \mathrm{p} \leq 0.01)$. Mientras la internalización se correlacionó con capital humano $(r=0.862 ; \mathrm{p} \leq 0.01)$, capital estructural $(r=0.880 ; \mathrm{p} \leq 0.01)$ y capital relacional $(r=0.824 ; \mathrm{p} \leq 0.01)$ con valores altos (cuadro 2). Dichos resultados fueron suficientes para soportar $H_{1}$.

CUADRO 2

CORRELACIÓN DE COMPONENTES

\begin{tabular}{|c|c|c|c|c|c|c|c|}
\hline Componentes & 1 & 2 & 3 & 4 & 5 & 6 & 7 \\
\hline Socialización & 1 & & & & & & \\
\hline Externalización & $0.829 * *$ & 1 & & & & & \\
\hline Combinación & $0.813^{* *}$ & $0.956^{* *}$ & 1 & & & & \\
\hline Internalización & $0.853^{* *}$ & $0.862^{* *}$ & $.856^{* *}$ & 1 & & & \\
\hline Capital Humano & $0.835^{* *}$ & $0.857^{* *}$ & $.769^{* *}$ & $.862^{* *}$ & 1 & & \\
\hline Capital Estructural & $0.815^{* *}$ & $0.867^{* *}$ & $.837 * *$ & $.880^{* *}$ & $.894 * *$ & 1 & \\
\hline Capital Relacional & $0.693^{* *}$ & $0.830^{* *}$ & $.772 * *$ & $.824^{* *}$ & $.902^{* * *}$ & $.922 * *$ & 1 \\
\hline
\end{tabular}

**. La correlación es significativa en un nivel 0.01 (bilateral) Fuente: Elaboración propia.

Como primer intento para poner a prueba las hipótesis $\mathrm{H}_{2}, \mathrm{H}_{3}$ y $\mathrm{H}_{4}$, es decir, corroborar el efecto de la gestión del conocimiento sobre el capital intelectual se realizó un análisis de regresión múltiple. El análisis de regresión múltiple estudio la relación de una variable dependiente con dos o más variables independientes (Anderson, Sweeney \& Williams, 2012). Las cuatro dimensiones de la gestión del conocimiento (socialización, externalización, combinación e internalización) ayudaron a explicar el $86 \%$ de la varianza del capital humano $\left(\mathrm{R}^{2}=0.861 ; \mathrm{F}=69.86\right), 82 \%$ del capital estructural $\left(\mathrm{R}^{2}=0.861 ; \mathrm{F}=52.509\right)$ y $76 \%$ del capital relacional $\left(\mathrm{R}^{2}=0.72 ; \mathrm{F}=35.72\right)$. Se encontró que tres componentes de gestión del conocimiento influyen de manera positiva y significativa sobre el capital humano: socialización ( $\beta=0.251 ; p \leq 0.05)$, externalización $(\beta=0.726$; $\mathrm{p} \leq 0.05)$ e internalización $(\beta=0.392 ; \mathrm{p} \leq 0.05)$, mientras que fue negativa en combinación $(\beta=-0.592 ; p \leq 0.05) \quad$ (cuadro 3). Tal evidencia empírica fue suficiente para soportar $\mathrm{H}_{2}$.

Por otro lado, sólo la internalización influyó sobre el capital estructural $(\beta=0.433$; $\mathrm{p} \leq 0.05)$; mientras que externalización $(\beta=0.653$; $\mathrm{p} \leq 0.05)$ e internalización $(\beta=0.543 ; \mathrm{p} \leq 0.05)$ influyeron de manera significativa y positiva sobre el capital relacional. Dicha evidencia empírica muestra que la influencia significativa de la gestión del conocimiento sobre el capital intelectual es limitada. Sobre el capital humano es completa; sin embargo, sólo influye la internalización sobre el capital estructural y, tanto la externalización como la internalización sobre el capital relacional. A través de lo anterior se observa que la evidencia no sustentó de manera completa $H_{3}$ y $H_{4}$. 
CUADRO 3

REGRESIÓN DE GESTIÓN DEL CONOCIMIENTO Y CAPITAL INTELECTUAL

\begin{tabular}{cccc}
\hline \multirow{2}{*}{ Gestión del conocimiento (VI) } & \multicolumn{3}{c}{ Capital intelectual (VD) } \\
\cline { 2 - 4 } & Capital humano & Capital estructural & Capital relacional \\
\hline Socialización (SO) & $0.251^{*}$ & 0.106 & -0.225 \\
Externalización (EX) & $0.726^{*}$ & 0.342 & $0.653^{*}$ \\
Combinación (CO) & $-0.592^{*}$ & -0.079 & -0.135 \\
Internalización (IN) & $0.392^{*}$ & $0.433^{*}$ & $0.543^{*}$ \\
$\mathrm{R}^{2}$ & 0.861 & 0.824 & 0.76 \\
$\mathrm{R}^{2}$ ajustada & 0.849 & 0.808 & 0.739 \\
$\mathrm{~F}$ & 69.86 & 52.509 & 35.72 \\
\hline
\end{tabular}

*Significativa en un nivel $\leq 0.05$

Fuente: Elaboración propia.

Con el fin de confirmar los resultados encontrados por medio de la regresión múltiple se elaboraron regresiones jerárquicas mediante la introducción de variables control (variables sociodemográficas: tamaño de la empresa, ubicación y área de comercialización). El análisis de regresión jerárquica busca detectar los efectos principales $y$ de interacción entre las variables de estudio (Cohen \& Cohen, 1983); mientras que la justificación del uso de variables control se sustenta en la búsqueda de evitar relaciones espurias (Wooldridge, 2000).
Por medio de la introducción de las variables control se localizó un efecto directo, significativo $y$ positivo sobre el capital humano por parte de los componentes de la gestión del conocimiento: socialización $\left(\beta=.702, \Delta \mathrm{R}^{2}=.346\right.$, $\mathrm{p}<0.001)$, externalización $\left(\beta=.845, \Delta \mathrm{R}^{2}=.346\right.$, $\mathrm{p}<0.001)$, combinación $\left(\beta=.837, \Delta \mathrm{R}^{2}=.219\right.$, $\mathrm{p}<$ $0.001)$ e internalización $\left(\beta=.750, \Delta \mathrm{R}^{2}=.372, \mathrm{p}<\right.$ 0.001 ) (cuadro 4). Cabe resaltar que la introducción de variables control mejoró el efecto directo de las variables de estudio en cuanto al nivel de significancia. 
CUADRO 4

REGRESIÓN JERÁRQUICA TENIENDO COMO VD (CAPITAL HUMANO)

\begin{tabular}{|c|c|c|c|c|}
\hline & \multirow[b]{2}{*}{ Paso predictor } & \multicolumn{3}{|c|}{ Capital Humano (VD) } \\
\hline & & Beta & $\Delta \mathrm{R}^{2}$ & Cambio en $\mathrm{F}$ \\
\hline & $\begin{array}{l}\text { Variables de control } \\
\text { Tamaño de la empresa } \\
\text { Ubicación } \\
\text { Área de comercialización }\end{array}$ & $\begin{array}{l}.093 \\
.127 \\
.083 \\
\end{array}$ & .395 & $10.026^{* *}$ \\
\hline \multirow[t]{2}{*}{2} & Socialización (SO) & $.702^{* * *}$ & .346 & $60.356^{* * *}$ \\
\hline & Paso predictor & Beta & $\Delta \mathrm{R}^{2}$ & Cambio en $\mathrm{F}$ \\
\hline 1 & $\begin{array}{l}\text { Variables de control } \\
\text { Tamaño de la empresa } \\
\text { Ubicación } \\
\text { Área de comercialización }\end{array}$ & $\begin{array}{c}-.025 \\
-.039 \\
.102 \\
\end{array}$ & .395 & $10.026^{* *}$ \\
\hline 2 & Externalización (EX) & $.845^{* * *}$ & .345 & $59.745^{* * *}$ \\
\hline 1 & $\begin{array}{l}\text { Variables de control } \\
\text { Tamaño de la empresa } \\
\text { Ubicación } \\
\text { Área de comercialización }\end{array}$ & $\begin{array}{l}.100 \\
.064 \\
.080\end{array}$ & .395 & $10.026^{*}$ \\
\hline \multirow[t]{2}{*}{2} & Combinación (CO) & $.637 * * *$ & .219 & $25.645^{* * * *}$ \\
\hline & Paso predictor & Beta & $\Delta \mathrm{R}^{2}$ & Cambio en $\mathrm{F}$ \\
\hline 1 & $\begin{array}{l}\text { Variables de control } \\
\text { Tamaño de la empresa } \\
\text { Ubicación } \\
\text { Área de comercialización }\end{array}$ & $\begin{array}{l}.089 \\
.107 \\
.036\end{array}$ & .395 & $10.026^{* *}$ \\
\hline 2 & Internalización (IN) & $0.750^{* * *}$ & .372 & $71.756^{* * *}$ \\
\hline
\end{tabular}

$* * \mathrm{p}<0.01 ; * * * \mathrm{p}<0.001$

Fuente: Elaboración propia.

Asimismo, se encontró que el capital estructural es afectado por la socialización $\left(\beta=.598, \Delta \mathrm{R}^{2}=.252, \mathrm{p}<0.001\right)$, externalización $\left(\beta=.733, \Delta \mathrm{R}^{2}=.260, \mathrm{p}<0.001\right)$, combinación $\left(\beta=.655, \Delta \mathrm{R}^{2}=.232, \mathrm{p}<0.001\right) \mathrm{e}$ internalización $\left(\beta=.693, \mathrm{DR}^{2}=.318, \mathrm{p}<0.001\right)$. Dichos resultados superan a los encontrados antes de la introducción de las variables control, donde sólo la internalización mostró un efecto sobre VD. En este caso, incluso las variables control tamaño de la muestra obtuvieron valores significativos respecto a SO $(\beta=.268, \mathrm{p}<0.01), \mathrm{CO}(\beta=.237, \mathrm{p}<0.01)$, e IN $(\beta=.249, \mathrm{p}<0.01)$ (cuadro 5).
En la tabla 6 se muestra un efecto positivo y significativo sobre el capital relacional por parte de SO $\left(\beta=.427, \Delta \mathrm{R}^{2}=.128\right.$, $\mathrm{p}<0.001), \quad \operatorname{EX}\left(\beta=.670, \mathrm{DR}^{2}=.217, \quad \mathrm{p}<0.001\right)$, $\operatorname{CO}\left(\beta=.541, \Delta \mathrm{R}^{2}=.159, \quad \mathrm{p}<0.001\right)$ e IN $(\beta=.604$, $\left.\Delta \mathrm{R}^{2}=.241, \mathrm{p}<0.001\right)$. Del mismo modo se obtuvieron resultados significativos de variables control como el tamaño de la empresa sobre SO $(\beta=.255, \mathrm{p}<0.01) y$ área de comercialización respecto a SO $(\beta=.278, \quad \mathrm{p}<0.01)$, EX $\quad(\beta=.286, \quad p<0.01), \quad(\beta=.267, \quad p<0.01)$ e IN $(\beta=.233, p<0.01)$. A través de los hallazgos encontrados en las regresiones jerárquicas por medio de la introducción de variables control se pudo soportar de manera completa $H_{2}, H_{3}$ y $H_{4}$. 


\section{CUADRO 5 \\ REGRESIÓN JERÁRQUICA TENIENDO COMO VD (CAPITAL ESTRUCTURAL)}

\begin{tabular}{|c|c|c|c|c|}
\hline & \multirow[b]{2}{*}{ Paso predictor } & \multicolumn{3}{|c|}{ Capital Estructural (VD) } \\
\hline & & Beta & $\Delta \mathrm{R}^{2}$ & Cambio en $\mathrm{F}$ \\
\hline & $\begin{array}{l}\text { Variables de control } \\
\text { Tamaño de la empresa } \\
\text { Ubicación } \\
\text { Área de comercialización }\end{array}$ & $\begin{array}{c}.268 * * \\
.104 \\
.109\end{array}$ & .534 & $17.544^{* *}$ \\
\hline \multirow[t]{2}{*}{2} & Socialización (SO) & $.598^{* * * *}$ & .252 & $52.812^{* * *}$ \\
\hline & Paso predictor & Beta & $\Delta \mathrm{R}^{2}$ & Cambio en $\mathrm{F}$ \\
\hline 1 & $\begin{array}{l}\text { Variables de control } \\
\text { Tamaño de la empresa } \\
\text { Ubicación } \\
\text { Área de comercialización }\end{array}$ & $\begin{array}{c}.163 \\
-.044 \\
.126 \\
\end{array}$ & .534 & $17.544^{* *}$ \\
\hline \multirow[t]{2}{*}{2} & Externalización (EX) & $.733^{* * *}$ & .260 & $56.520 * * *$ \\
\hline & Paso predictor & Beta & $\Delta \mathrm{R}^{2}$ & Cambio en $\mathrm{F}$ \\
\hline 1 & $\begin{array}{l}\text { Variables de control } \\
\text { Tamaño de la empresa } \\
\text { Ubicación } \\
\text { Área de comercialización }\end{array}$ & $\begin{array}{l}.237^{* *} \\
.003 \\
.100 \\
\end{array}$ & .534 & $17.544^{* *}$ \\
\hline \multirow[t]{2}{*}{2} & Combinación (CO) & $.655^{* * *}$ & .232 & $44.661^{* * *}$ \\
\hline & Paso predictor & Beta & $\Delta \mathrm{R}^{2}$ & Cambio en $\mathrm{F}$ \\
\hline 1 & $\begin{array}{l}\text { Variables de control } \\
\text { Tamaño de la empresa } \\
\text { Ubicación } \\
\text { Área de comercialización }\end{array}$ & $\begin{array}{l}.249 * * \\
.071 \\
.064 \\
\end{array}$ & .534 & $17.544^{* *}$ \\
\hline 2 & Internalización (IN) & $0.693^{* * *}$ & .318 & $96.306^{* * *}$ \\
\hline
\end{tabular}

$* * \mathrm{p}<0.01 ; * * * \mathrm{p}<0.001$.

Fuente: Elaboración propia. 


\section{CUADRO 6 \\ REGRESIÓN JERÁRQUICA TENIENDO COMO VD (CAPITAL RELACIONAL)}

\begin{tabular}{|c|c|c|c|c|}
\hline & \multirow[b]{2}{*}{ Paso predictor } & \multicolumn{3}{|c|}{ Capital Relacional (VD) } \\
\hline & & Beta & $\Delta \mathrm{R}^{2}$ & Cambio en $\mathrm{F}$ \\
\hline 1 & $\begin{array}{l}\text { Variables de control } \\
\text { Tamaño de la empresa } \\
\text { Ubicación } \\
\text { Área de comercialización }\end{array}$ & $\begin{array}{l}.255^{*} \\
.076 \\
.278^{*}\end{array}$ & .561 & $19.556^{*}$ \\
\hline \multirow[t]{2}{*}{2} & Socialización (SO) & $.427^{* * *}$ & .128 & $18.513^{* * *}$ \\
\hline & Paso predictor & Beta & $\Delta \mathrm{R}^{2}$ & Cambio en $\mathrm{F}$ \\
\hline 1 & $\begin{array}{l}\text { Variables de control } \\
\text { Tamaño de la empresa } \\
\text { Ubicación } \\
\text { Área de comercialización }\end{array}$ & $\begin{array}{c}.121 \\
-.093 \\
.286^{* *}\end{array}$ & .561 & $19.556^{*}$ \\
\hline \multirow[t]{2}{*}{2} & Externalización (EX) & $.670^{* * *}$ & .217 & $43.948^{* * *}$ \\
\hline & Paso predictor & Beta & $\Delta \mathrm{R}^{2}$ & Cambio en $\mathrm{F}$ \\
\hline 1 & $\begin{array}{l}\text { Variables de control } \\
\text { Tamaño de la empresa } \\
\text { Ubicación } \\
\text { Área de comercialización }\end{array}$ & $\begin{array}{l}.208 \\
-.026 \\
.267^{*}\end{array}$ & .561 & $19.556^{*}$ \\
\hline \multirow[t]{2}{*}{2} & Combinación (CO) & $.541^{* * *}$ & .159 & $25.448^{* * *}$ \\
\hline & Paso predictor & Beta & $\Delta \mathrm{R}^{2}$ & Cambio en $\mathrm{F}$ \\
\hline 1 & $\begin{array}{l}\text { Variables de control } \\
\text { Tamaño de la empresa } \\
\text { Ubicación } \\
\text { Área de comercialización }\end{array}$ & $\begin{array}{l}.209^{*} \\
.021 \\
.233^{*}\end{array}$ & .561 & $19.556^{*}$ \\
\hline 2 & Internalización (IN) & $.604^{* * * *}$ & .241 & $54.657 * * *$ \\
\hline
\end{tabular}

$* \mathrm{p}<0.05 ; * * \mathrm{p}<0.01 ; * * * \mathrm{p}<0.001$.

Fuente: Elaboración propia. 


\section{CONCLUSIONES}

Dentro de la presente investigación se parte del hecho de la interrelación entre gestión del conocimiento y capital intelectual. La gestión del conocimiento es la función que planifica, coordina y controla los flujos de conocimientos que se producen en la empresa en relación con sus actividades y con su entorno (Bueno, 1999). El conocimiento como recurso intangible se encuentra inmerso dentro del capital intelectual. El capital humano formado por el conocimiento individual, su socialización, educación, desarrollo de habilidades, comunicación, capacitación y aprendizaje (Roos et al, 2001), conlleva el empleo del conocimiento. Asimismo, el capital estructural por medio de los sistemas, uso de tecnología, infraestructura, organización, investigación y procesos (CIC, 2002), convierte la información en conocimiento. Mientras que el capital relacional, como la forma de interactuar con los clientes, proveedores, otras empresas, instituciones públicas y organizaciones sociales (CIC, 2003), es determinado por el conocimiento creado, transformado y transmitido dentro de la empresa.

El objetivo de la presente investigación es conocer el grado de asociación de la gestión del conocimiento y el capital intelectual en la industria tequilera del estado de Jalisco. A través de una investigación de tipo cuantitativa $y$ correlacional, con un diseño no experimental fue posible sustentar empíricamente todas las hipótesis planteadas.

La evidencia empírica encontrada contribuye a la teoría de recursos y capacidades (RBV) $y$ a la teoría basada en el conocimiento (KBV). Referente a la primera se observa que el conocimiento es un recurso intangible capaz de darle a la organización una ventaja competitiva, al ser raro, valioso, imperfectamente imitable y no sustituible (Barney, 1991). En KBV se contem- pla a la gestión del conocimiento como elemento central para el desarrollo de capacidades en las organizaciones (Grant, 1996a), que se fundamenta en el proceso de transferencia, capacidad de absorción, apropiación, especialización y transformación del conocimiento en productos dentro de la empresa (Grant, 1996b). Mediante los hallazgos se encuentra al capital intelectual como un recurso que se asocia y es afectado por la gestión del conocimiento.

Si bien existen muchas menciones sobre relación entre las variables de estudio, se destaca la necesidad de evidencia empírica que sustente tal postura. A través de una muestra de 50 empresas tequileras del Estado de Jalisco se localiza evidencia empírica que sostiene la asociación entre las variables de estudio, tal como proponen Koening (1998), Bueno (1999), Vargas, (2002), Martínez, (2003), Curado (2008), Booker, Bontis y Serenko (2008); Antosova y Csikosova, (2011) Serenko, Bontis y Hull (2011), Salmninezhad y Daneshvar (2012), Fierro, Mercado y Cernas (2012). Se observa que los componentes de la gestión del conocimiento (socialización, externalización, combinación e internalización) se asociaron de manera positiva $y$ significativa con el capital intelectual (capital humano, capital estructural y capital relacional).

Es cierto que en un primer momento, al tratar de corroborar si la gestión del conocimiento influye sobre el capital intelectual se puso a prueba , y mediante regresión múltiple, los hallazgos mostraron que la gestión del conocimiento influye de forma positiva y significativa sobre el capital humano por medio de la socialización, externalización e internalización; mientras que en el caso de la combinación la influencia fue negativa. Para el capital estructural y relacional el nivel de influencia fue limitado. En el primero la influencia fue exclusiva para la internalización; mientras que para el capital relacional sólo se observó en la externa- 
lización e internalización. Tal evidencia empírica mostró que la gestión del conocimiento es de forma parcial una variable predictora del capital intelectual, especialmente del capital humano; sin embargo, no soporta del todo lo propuesto por Fierro, Mercado y Cernas (2012).

En la introducción de variables control como el tamaño de la empresa, ubicación y área de comercialización con el fin de corroborar los resultados de la regresión múltiple fue posible encontrar una mejoría en el efecto de la variable independiente sobre la dependiente, en el entendimiento de que la gestión del conocimiento es un proceso y el capital intelectual es su producto (Fierro, Mercado \& Cernas, 2012). Se observó que todos los componentes de gestión del conocimiento influyen de manera positiva $y$ significativa sobre el capital intelectual. Tales hallazgos corroboran la existencia de un efecto real, ya que la variable independiente afecta de manera directa la dependiente sin la intervención y con la intervención de variables control (Wooldridge, 2000). La presente evidencia empírica soporta las posturas de Bueno (1999), Marr et al (2003) y Fierro, Mercado y Cernas (2012).

A través de los presentes hallazgos se puede interpretar que el éxito de la gestión del conocimiento implica el desarrollo del capital intelectual (Marr et al, 2003), la gestión del conocimiento integra el capital humano, el cual es un valor del conocimiento creado por las personas; administra el capital estructural, conocimiento creado en la organización materializado en sistemas, procedimientos y tecnología; y desarrolla el capital relacional, conocimiento creado en relación con su entorno (Bueno, 1999). Además, la gestión del conocimiento desarrolla al conocimiento tácito $y$ explícito a través de procesos pertenecientes al capital humano; permite su combinación a través de procesos sociales dentro del capital estructural; $y$, se obtiene, incorpora $y$ aplica por medio de la relación con clientes, proveedores, académicos, agentes de mercado y proveedores, es decir por medio del capital relacional (Fierro, Mercado \& Cernas, 2012).

Dentro de la regresión jerárquica se observa que la variable control referente al tamaño de la empresa obtuvo valores significativos sobre el capital estructural y relacional, así como el área de comercialización respecto al capital relacional. Dichos resultados sólo pueden ser entendidos desde el contexto de la industria de estudio. A partir de los años noventa se empezó a vislumbrar diferencia de competitividad dentro de la industria, pues empresas como Cuervo, Herradura, Sauza y Cazadores ya comenzaban a tener una participación del mercado de manera sobresaliente (Macías, 1997), las cuales para el 2004 ya ocupaban el 65\% el mercado (Coelho \& Castillo, 2004). De hecho, la industria del tequila se caracteriza como un oligopolio (Macías, 2001), donde la mayoría de las empresas tequileras son familiares y se encuentran estancadas en técnicas ancestrales que impiden la competitividad que demanda la cadena de esta industria (Salvador \& Maldonado, 2010).

A partir de lo anterior se puede observar una participación relevante del tamaño de la empresa y el área de comercialización para que sea patente el efecto de la gestión de conocimiento sobre el capital intelectual dentro tal industria. Dentro del capital estructural tal fenómeno se observa en el hecho de que el desarrollo tecnológico, innovación, infraestructura e investigación son promovidos por las empresas líderes (Coelho \& Castillo, 2004). Asimismo, la carencia en asesoría técnica, falta de información y baja productividad (Luna, 2001), sólo pueden ser percibidos al contemplar el tamaño de las empresas. En el capital relacional la industria tiene una dependencia de capitales extranjeros, la cual afecta en las relaciones con productores de agave, papel estratégico de las marcas, relaciones con el mercado y acceso al mercado internacional (Coelho, 2007), donde el tamaño de la empresa y su área de comercialización muestran la diferenciación entre los posibles efectos de la variable independiente sobre la dependiente.

Del mismo modo, una posible explicación de los resultados presentados es que algunas de las empresas tequileras presentan problemas respecto a la creación y transferencia tecnológica, falta de investigación y desarrollo, poca relación con centros académicos, medios de comunicación inadecuados y escasos proyectos de investigación (COFUPRO, 2003), lo cual 
puede se debe en parte por el dominio de las cuatro empresas líderes en manos de capitales extranjeros (Macías \& Valenzuela, 2009).

De tal modo, los hallazgos encontrados sólo pueden ser interpretados a la luz del contexto de la industria. La evidencia empírica obtenida refleja la necesidad de la consideración de otra filosofía en las empresas tequileras de Jalisco (Díaz, 2007), lo cual les permita alcanzar mayor competitividad, donde activos intangibles como la gestión del conocimiento $y$ capital intelectual puedan ser un plus para estas empresas.

La regresión jerárquica muestra un efecto positivo y significativo que sólo pudo ser observado por medio de la intervención de variables control, por lo cual para próximas investigaciones se requiere abordar a dicha industria dentro de su complejidad contextual, donde se permita la participación de otras variables sociodemográficas que faciliten el hallazgo de evidencia práctica que sustente la aplicación de postulados teóricos.

Una limitación del estudio fue la utilización exclusiva de un instrumento de medición cuantitativo; sin embargo, donde la principal debilidad se encuentra el tamaño de la muestra obtenida y su tipo de muestreo no probabilístico. No obstante próximas líneas de investigación pueden dar a conocer evidencia empírica en otros países y con otro tipo de organización, donde se pueda tener una muestra mayor. Para próximas investigaciones sería conveniente obtener otra muestra en la misma industria, donde se permita realizar un estudio con diseño longitudinal, con el fin de medir a través del tiempo cómo influye de la gestión del conocimiento sobre el capital intelectual a través del tiempo.

\section{REFERENCIAS BIBLIOGRÁFICAS}

Andersen, A. (1999). El Management en el Siglo XXI. Granica. Buenos Aires.

Anderson, D. R., Sweeney, D. J. \& Williams, T. (2012). Estadística para negocios y economía. (11ª. Ed.). México: Cengage Learning.

Angulo, E., \& Negron, M. (2008). Modelo holísitoc par ala gestión del conocimiento.
Scientific e-journal of Management Science. 11 (4): 38-51.

Antosova, M. \& Csikosova, A. (2011). Intellectual capital in context of knowledge management, En Pachura, P. (Ed.) The Economic Geography of Globalization. Disponible en http://www. intechopen.com/books/the-economicgeography-ofglobalization/intellectualcapital-incontext-of-knowledge-management

Barney, J. (1991). Firm resources and sustained competitive advantage. Journal of Management 17: 99-120.

Barney, J. B., Ketchen, D., \& Wright, M. (2011). The future of Resource-Based Theory: Revitalization or Decline? Journal of Management. 37 (5): 1299-1315.

Bontis, N. (1996). Intellectual Capital: an exploratory study that develops measures and models. Management Decision, 36 (2): 67-76.

Bontis, N. (1999). Managinf Organizational Knowledge by diagnosing intellectual capital: Framing and advancing the state of the Field. International Journal Technology Management, 18: 433-462.

Bratianu, C. (2010). A critical analysis of Nanaka's model of knowlegde dinamics. Electronic Journal of Knowledge Management. 8 (2): 193-200

Booker, L., Bontis, N., Serenko, A. (2008). The relevance of knowledge management and inellectual capial research. Knowledge Management and Process Management. 15: 235-246.

Brooking, A. (1997a). El capital intelectual, el principal activo de las empresas del tercer milenio. Paidós. Barcelona.

Brooking, A. (1997b). The management of intellectual capital. Long Range Planning, 30 (3): 364-365.

Bueno, E. (1999). La gestión del Conocimiento: Nuevos perfiles profesionales. Boletín de Estudios Económicos. 3 (164): 207-229.

Casas-Campillo, C. (1991). La Tercera Revolución Industrial en México. Alternativas de la Industria de Fermentaciones en México (pp. 37-43). 
Instituo de Investigaciones Económicas, UNAM. México.

CIC. (2002). Identificación y Medición del Capital Relacional. Documentos Intellectus, Centro de Investigación sobre la Sociedad del Conocimiento (No. 2). España.

CIC. (2003). Modelo Intellectus: Medición y Gestión del Capital Intelectual. (CICIADE, Ed.) Documentos Intellectus (No. 5). España.

Coelho, A. \& Castillo. V. (2004). Firms and international governance in tequila cluster. In Memorias, Congreso Internacional Agroindustria Rural y Territorio (ARTE), 1-4 Diciembre 2014. GIS SYAL. México.

Coelho, A. (2007). Eficiencia Colectiva y Upgranding en el Cluster del Tequila. Revista Análisis Económico, 22 (49): 169-194.

COFUPRO. (2003). Caracterización de la cadena agroalimentaria/agroindustrial nacional e identificación de sus demandas tecnológicas. Programa Nacional Estratégico de Necidades de Investigación y de Transferencia de Tecnología. México.

Cohen J. \& Cohen P., (1983) Applied Multiple Regression. Correlation Analysis for the Behavioral Sciences (2da ed.). New Jersey: Erlbaum.

CONSEJO REGULADOR DEL TEQUILA (2013). Estadísticas. Enero. Disponible en www. crt.org.mx. (Enero de 2013).

Cortés Marató, J., \& Martínez Riu, A. (1996). Diccionario de filosofía en CD-ROM. Barcelona: Empresa Editorial Herder.

Curado, C. (2008). Perceptions of knowledge management and intellectual capital in the banking industry. Journal of Knowledge Management. 12 (3): 141-155.

Díaz, O. H. (2007). La productividad de las empresas fabricantes de tequila en Jalisco. Carta Económica Regional. 19 (99): 40-45.

Drucker, P. F. (1969). The age of discontinuity: Guidelines to our changing society. Harper \& Row. NY.

Edvinsson, L. (1997). Developing intellectual capital at Skandia. Long Range Planning. 30 (3): 366-373.
Edvinsson, L., \& Malone, M. S. (2001). El capital Intelectual, Cómo identificar y calcular el valor de los recursos intangibles de su empresa. Gestión 2000. México.

Edwars, J., Collier, M., \& Shaw, D. (2005). Knowledge Management and its impact on the Management Accountant. Aston Business School, Aston University. London.

Fierro, E., Mercado, P., \& Cernas, D. A. (2012). Gestión del conocimiento y capital intelectual desde la teoría de recursos y capacidades. En P. Mercado, \& D. A. Cernas, El capital intelectual en las organizaciones. Una agenda de investigación (4555). Bonobos Editores. Toluca.

Funes, Y. (2010). Valuación de los activos intangibles. Caso de la UNAM. Revista del Centro de Investigación. 9 (33): 45-60

Grant, R. M. (1996a). Toward a knowledgebased theory of the firm. Strategic Management Journal. 17: 109-122.

Grant, R. M. (1996b). Prospering in dynamically-competitive enviroments: organizational capability as knowledge integration. Organizational Science. 7 (4): 375-387.

Hair F., Anderson R, Black W. (2004). Análisis multivariante, (5a ed.) Prentice Hall. España.

Kaplan, R., \& Norton, D. (1992). Balanced Scorecard, Measures that drive Performance. Harvard Business Review January-february: 70-79.

Koening, M. E. (1998). From intelectual capital to knowledge management: what are they talking about? INSPEL. 32 (4): 222 233.

Kogut, R., \& Zander, U. (1992). Knowledgge of the firm, combinate capabilities and the replication of the technology. Organization Science. 3 (3): 383-397.

Luna, R. (2001). Mestizaje y el Tequila. Sincronía, Primavera. Disponible en: http://sincronia.cucsh.udg.mx/mestiz. htm

Macías, A. (1997). Organziación de la Industria del Tequila. Carta Económica Regional. 9 (54). 3-10. 
Macías, A. (2001). El cluster en la Industria del Tequila en Jalisco, México. Agroalimentaria, 13 (13).

Macías, A., \& Valenzuela, A. G. (2009). El tequila en tiempos de la mundialización. Comercio Exterior. 50 (6): 459-471.

Machlup, F. (1962). The production and distribution of knowledge in the United States. Princeton University Press. New Jersey.

Mantilla, S. A. (2000). Capital intelectual, Contabilidad del Conocimiento (2a. ed.). Ecoe Ediciones. Bogotá.

Martínez, M.: (2003). De la contabilidad de los recursos humanos al capital intelectual y la gestión del conocimiento: una ampliación necesaria. Direccion y organizacion: Revista de dirección, organización y administración de empresas. Julio 29: 134-144.

Marr, B. \& Schiuma, G. (2001). Measuring and managing intellectual capital and knowledge assets in new economy organisations, In Bourne, M. (Ed.), Handbook of Performance Measument. Lee. London:.

Marr, B., Gupta, O., Pike, S. \& Roos, G. (2003). Intellectual capital and knowledge management effectiveness. Management Decision. 41 (8): 771-781.

Mercado, P., \& Cernas, D. A. (2012). El capital intelectual en las organizaciones. Una agenda de investigación. Bonobos Editores. México.

Mercado, P., Cernas, D.A. \& Demuner, M. R., (2013). Construcción y validez de una escala de capital intelectual para instituciones públicas de educación superior. En dictamen. UAEMex: México.

Mihi, A., García, V. J., \& Rojas, R. M. (2011). Knowledge creation, organization learning and ther effects on organizational performance. Inzinerine EkonomikaEngineering Economics. 3 (22): 309-318.

Montoya S. O. (2007) Evaluación de confiabilidad $y$ validez de una versión en español del cuestionario breve impacto del peso en la calidad de vida en pacientes de la clínica Reina Sofía en Bogotá años 20062008, Rev.Medica.Sanitas. 12 (3): 18-35

Nevado, D., \& López, V. (2002). El capital Intelectual. Modelos, informes, desarrollos y aplicaciones. Valoración y Medición. Prentince Hall: Madrid.

Nonaka, I. (1988). Toward Middle-Up Down Management: Accelerating information creation. Sloan Management Review , Vol. 29 (No. 3), pp. 9-18.

Nonaka, I. (1991). The knowledge-creating company. Harvard Business Review , pp. 96-104.

Nonaka, I. (1994). A dynamic theory of knowledge creation. Organizational Science. 5 (1): 14-37.

Nonaka, I., \& Konno, N. (1994). The concept of Ba: Building Knowledge Creation. Califorornia Management Review. 40 (3): 14-37.

Nonaka, I., \& Takeuchi, H. (1995). The Knowledge-Creating Company. Oxford University Press. New York.

Nonaka, I., Reinmoeller, P., \& Senoo, D. (1998). Managment focus: the art of knowledge systems to capitalize market knowledge. European Management Journal. 16 (6): 673-684.

Nonaka, I., \& Teece, D. (1999). Managing industrial knowledge: Creation, transfer and utilization. SAGE Publications. U.S.

Nonaka, I., \& Toyama, R. (2003). The knowledge-creating theory revisted: knowledge creatin as a synthesizing process. Knowledge Managamente Research \& Practice. 1: 2-10.

Nonaka, I. (2007). La empresa creadora de conocimiento. Harvard Business Review: julia: 1-9.

Madrigal, B. E. (2009). Capital humano e intelectual: su evaluación. Observatorio Laboral Revista Venezonala. 2 (3): 65-81.

Obeso, C. (2003). Capital intelectual. Ediciones Gestión 2000. Barcelona.

Ordoñez de Pablos, P. (2004). Capital intelectual, gestión del conocimiento y sistemas de gestión de recursos humanos: influencia sobre los resultados organizativos en la industria española. XIV Congreso $A C E D E$. ACEDE: Murcia.

Orozco, J. L. (2011). Las relaciones de poder en los intercambios comerciales de la cadena productiva del tequila y su incidencia 
de la competitividad de la rama industrial. Tlaquepaque: ITESO, Tesis doctoral en estudios científicos y sociales.

Penrose, E. T. (1959). The theory of growth of the firm. Wiley. New York.

Popper, K. (1978). La lógica de las ciencias sociales. Editorial Grijalbo. México.

Ramírez, Y. Loyduy, \& Rojas J. (2007). Intellectual capital management in spanish universities. Journal of Intellectual Capital. 8 (4): 732-748.

Requena, J. V., Sellens, J. T., \& Masllorens, J. L. (2003). Inversión en intangibles y competitividad internacional de la empresa española. Estudios de Economía Aplicada. 21 (3): 503-520.

Rodríguez Gómez, D. (2006). Modelos para la creación y gestión del conocimiento una aproximación teórica. Educar. 37: 25-39.

Roos, J., Roos, G., Dragonetti, N., \& Edvinsson, L. (2001). Capital Intelectual, El intangible de la empresa. Paidós. Barcelona.

Rulfo, V., \& et al. (2007). Conocimiento y prácticas agronómicas para la producción de Agave tequilana Weber en la zona de denominación de Origen del tequila. Jalisco: Instituto Nacional de Investigaciones Forestales, Agrícolas y Pecuarias. Centro de Investigación Regional del Pacífico Centro.

Saint-Onge, H. (1996). Tacit knowledge: the key to the strategic aligment of intellectual capital. Strategy and Leadership. 24 (2): 10-14.

Salmaninezhad, R. A. \& Daneshvar, M. (2012). Relationship analysis between intellectual capital and knowledge management (Case study: Tehran Science \& Technology Park). International Journal of Contemporary Research Business. 3 (10): 135-143.

Salvador, \& Maldonado (2010). El agave tequilero debe romper su letargo tecnológico. El informador. 09 de Noviembre. Guadalajara.
Serenko, A., Bontis, N., \& Hull, E. (2011). Practical relevance of knowledge management and intellectual capital scholarly research: Brooks as knowledge translation agents. Knowledge \& Process Management. 18 (1): 1-9.

Simó, P., \& Sallán, J. M. (2008). Capital Intangible y Capital Intelectual: Revisión, definiciones y líneas de investigación. Estudios de Economía Aplicada , Vol. 26 (No. 2), pp. 65-78.

Sullivan, P. (2001). Rentabilizar el Capital Intelectual, Técnicas para optimizar el valor de la Innovación. Ediciones Paidós Ibérica. España.

Skandia (1994). Visualizing intellectual capital in Skandia. Sweden, Intellectual capital supplement. Sweden.

Stewart, T. (1998). La nueva riqueza de las organizaciones: el capital intelectual. Ediciones Granica. Barcelona.

Sveiby, K. E. (1996). The intangible Assets Monitor. Journal of Human Resource Costing and Accounting. 2 (1): 73-97.

Tejedor, B., \& Aguirre, A. (1998). Proyecto Logos: Investigación relativa a la capacidad de aprender de las empresas españolas. Boletín de Estudios Económicos. 3 (164): 231-249.

Vargas, A. (2002). De la participación a la gestión del conocimiento $y$ del capital intelectual: Reflexiones en torno a la empresa cooperativa. CIRIEC-España, Revisata de Economía Pública, Social y Cooperativa, abril mayo. 40: 123-140.

Verneaux, R. (1994). Epistemología general o crítica del conocimiento. Herder. Barcelona.

Wooldridge, J. M. (2000). Introducción a la econometría . México: Thompson.

$\mathrm{Wu}, \mathrm{A}$. (2005). The Integrations between balanced scorecard and intellectual capital. Journal of Intellectual Capital. 2 (6): 267284. 